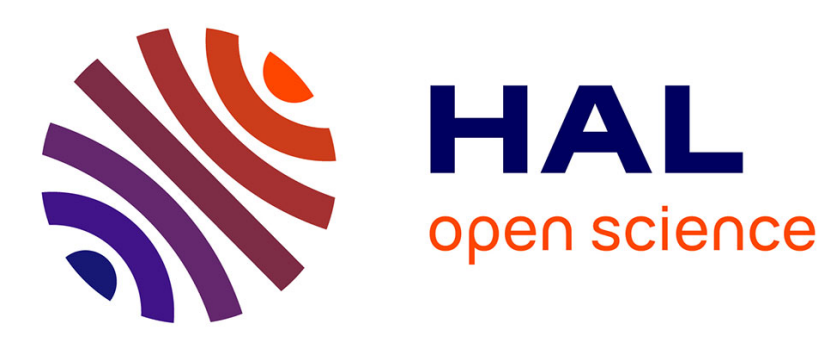

\title{
Geometric Calibration of an OCT Imaging System
}

\author{
Mouloud Ourak, Brahim Tamadazte, Guillaume Laurent, Nicolas Andreff
}

\section{To cite this version:}

Mouloud Ourak, Brahim Tamadazte, Guillaume Laurent, Nicolas Andreff. Geometric Calibration of an OCT Imaging System. International Conference on Robotics and Automation, May 2018, Brisbane, Australia. hal-02868021

\section{HAL Id: hal-02868021 \\ https://hal.science/hal-02868021}

Submitted on 15 Jun 2020

HAL is a multi-disciplinary open access archive for the deposit and dissemination of scientific research documents, whether they are published or not. The documents may come from teaching and research institutions in France or abroad, or from public or private research centers.
L'archive ouverte pluridisciplinaire HAL, est destinée au dépôt et à la diffusion de documents scientifiques de niveau recherche, publiés ou non, émanant des établissements d'enseignement et de recherche français ou étrangers, des laboratoires publics ou privés. 


\title{
Geometric Calibration of an OCT Imaging System
}

\author{
M. Ourak, B. Tamadazte, G. J. Laurent and N. Andreff
}

\begin{abstract}
This paper deals with an OCT (optical coherence tomography) geometric calibration method. OCT medical imaging system has received a growing interest during the last two decades. In medical purposes, OCT images are generally called optical biopsies which allows in-vivo investigation almost similar to a histopathological study. The physician can rely on the OCT images to establish a rapid and direct diagnosis. But the OCT images formation suffered numerous distortions due in particular to the complex optical path, from the source to the viewed sample passing through than two reflecting mirrors and a scan objective. The obtained optical biopsies include several spectral and geometric distortions. The proposed calibration model aims to compensate the distortions. More precisely, two models were developed allowing the correction of both 2D images (B-Scan slices) and 3D images (volume). These models were experimentally validated (in both artificial and biological samples) using a spectral domain OCT system. It has demonstrated a significant enhancement of the OCT images accuracy.
\end{abstract}

\section{INTRODUCTION}

The calibration of an optical imaging system consists of determining the mathematical relationship between an observed scene (e.g., 3D points) and its projection into a 2D image [1]. Camera calibration is a primordial step in several computer vision and robotics applications, e.g., 3D reconstruction, image-based metrology, robot vision control, object tracking and recognition, etc. Generally, a conventional calibration model includes two parts: intrinsic and extrinsic parameters. For instance, in case of pinhole camera, the first set of parameters describes the projective mapping from world coordinates to pixel ones while the second denotes the camera 3D pose [2].

The work described in this paper deals with a specific optical imaging system which is named OCT (optical coherence tomography). OCT images formation does not look like a convention projective camera which have benefited from very active research over the last 30-40 years. Indeed, OCT is operating under the principle of low coherence interferometry providing very interesting lateral and axial resolutions of a few micrometers. Depending on the intended use, OCT offers three types of optical biopsies 1D (A-Scan optical core), 2D (B-Scan image) and 3D (C-Scan volume). OCT imaging modality is able to reach higher penetration depths $1-5 \mathrm{~mm}$ compared to only $250 \mu \mathrm{m}$ provided by the confocal microscopy devices. Consequently, OCT modality

This work is conducted with a financial support from the project NEMRO (ANR-14-CE17-0013-001) funded by the Agence Nationale de la Recherche, France. It is also performed in the framework of the Labex ACTION (ANR-11-LABX-0001-01)

The authors are with FEMTO-ST institute, AS2M department, Univ. Bourgogne Franche-Comté/CNRS/ENSMM, 25000 Besano̧n, France. brahim.tamadaztedfemto-st.fr is highly suited, especially for imaging micron-scale internal structures.

Due the fact that OCT is a recent imaging modality with a specific use (initially in ophthalmology, dermatology and more recently in cardiology and few others medical fields), there is no much reported works relating to OCT calibration, despite a clear need to make more reliable optical biopsybased diagnosis or accurate OCT-guided robotic instruments during interventional tasks [3], [4]. The first calibration methods reported in the literature deal with spectral calibration dedicated to the computation of the mapping between the OCT interference signals and the corresponding (real) depths of the viewed sample. To get a realistic OCT image (with real depth), the operator uses a calibration template with wellknown depths to tune the spectral calibration parameters.

Westphal et al. [5] proposed a method to correct BScan OCT images, i.e., compensate the different 2D image geometric distortions. This method is based on the Fermat's optical principle to carry out a mathematical model of the image deformations caused by the scanning mechanism (a pair of galvanometric mirrors). The OCT images correction seems efficient, but the method is limited to a planar correction, i.e., 2D images. In the continuity of this work, Van der Jeught et al. [6] are interested in the geometric distortions in case of wide field-of-view OCT systems. This kind of systems suffer from the amplification of geometric deformation mainly on the image edges. Indeed, this is due to the high sweep angles required to cover the whole sample surface. The authors have proposed a deformation model based on a straightforward coordinate transformation scheme to tackle the problem of image artefacts. In order to optimize the computation time of their calibration algorithm, they chose a GPU (Graphics Processing Unit) implementation. More recently, in [7] it is suggested another method based on the use of well-known and accurate 3D pattern to perform the calibration process. In a certain way, the proposed approach is similar to the calibration methods established for the conventional projective cameras, i.e., the authors achieve the matching between the real 3D world points (template markers) to the $2 \mathrm{D}$ visual points extracted from the OCT images to compute the complete transformation between the image frame and the OCT frame. It can be underlined that the efficiency of this approach is strongly related to the accuracy of the used calibration template.

In our work, we focused in the entire (2D and 3D) calibration of the OCT system using a simple flat object (without any visual markers) placed on the sample platform. By detecting the object surface into several OCT images, it is possible to trace the different geometric distortions and thus 
compensate them accurately in the reconstructed images. The proposed analytical model has demonstrated his ability to correct both 2D and 3D OCT images. In particular, the developed approach allows compensating the three known geometric distortions, namely the spherical, the angular, the fan deformations listed in [8].

The paper is organized as follows: Section II discusses the OCT image distortion problem. The proposed calibration models for 2D and 3D OCT images correction are presented in Section III while Section IV describes the experimental validation set-up including the calibrated OCT system as well as the obtained intrinsic and extrinsic parameters of the considered OCT system.

\section{OCT IMAGE FORMING}
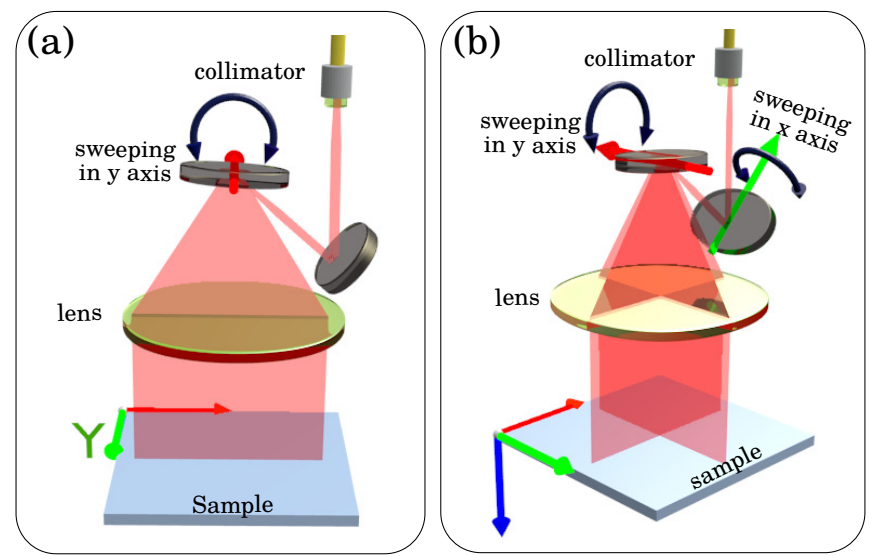

Fig. 1. Optical path: (a) one axis scanning mode (2D acquisition) and (b) two axes scanning mode (3D acquisition).

The working principle of an OCT system is similar to ultrasound imaging (US): it is based on the measure of the echo time delay and the intensity of the back-scattered or reflected light from the sample [9]. The time of flight of the light-wave provides information about the position of the scattering site in the sample, while the intensity of the backscattered light depends on the optical properties of the tissue. Since light travels faster than sound $\left(3 \times 10^{8} \mathrm{~m} / \mathrm{s}\right.$ for the light versus $1500 \mathrm{~m} / \mathrm{s}$ for the sound in water), direct measurement of the echo time as in US systems is not viable. In OCT systems, this measure is indirectly achieved by using low-coherence interferometry and implementing a Michelson interferometer. Light from a low coherence light beam is directed onto a beam splitter and split into reference and sample arms. The light in the reference arm is reflected by a mirror placed at a known distance, whereas the light in the sample arm is focused on the sample by a scanning mechanism designed to scan the focal spot in lateral directions. The light back-scattered or reflected from the sample is interfered with the returning reference arm light and travels to the spectrometer where the unique phase delay for each wavelength is detected. The depth information is acquired using a Fast Fourier Transform (FFT). The geometric distortions mentioned in the introduction are essentially due to the optical scanning system composed of two galvanometer actuated mirrors and a telecentric objective that allow the scanning in two axes.

Depending on the intended use, OCT offers three types of outputs: A-Scans ( $z$ optical cores), B-Scans ( $x z$ or $y z$ images as illustrated in Fig. 1(a)) and C-Scan (xyz 3D images as illustrated in Fig. 1(b)). As example, Fig. 2(a) shows the OCT probe placed above a sample holder, when Fig. 2(b) depicts some examples of grabbed 3D OCT images of a flat object illustrating different geometric distortions.
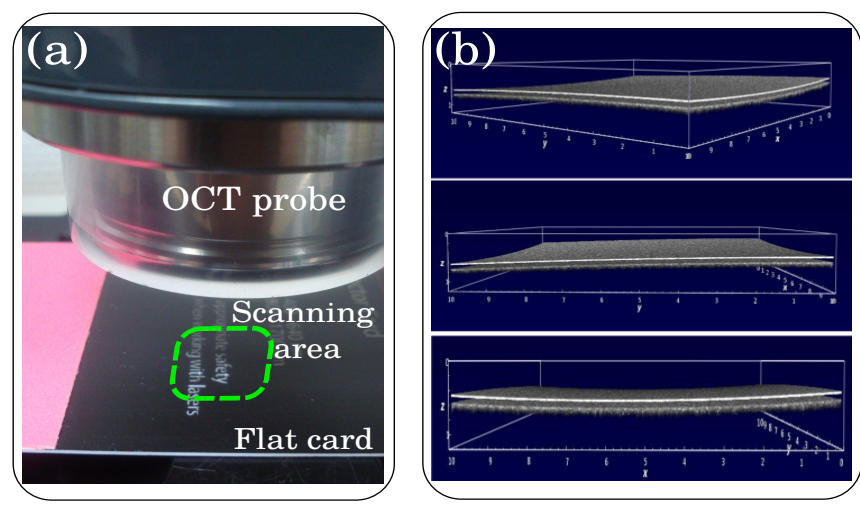

Fig. 2. Illustration of the geometric distortions in case of 3D OCT image acquisition: (a) shows the area to be scanned and (b) the acquired 3D OCT image seen from several angles. Also, (b) depicts the the result of the distortions effects on the grabbed OCT images.

As can be seen in Fig. 2(b), the OCT device views the sample as curved surfaces instead of planar and horizontal ones (note that the sample is calibration template perfectly planar provided by THORLABS). This is due to the geometric distortions which characterize most OCT systems. Consequently, using optical biopsies to perform a reliable diagnosis, by relying on the size, position, topology of the structural aspect of the sample, becomes difficult. It is therefore necessary to make compensation to these optical deformations in aim to get optical biopsies reflecting accurately the reality of the observed sample.

\section{2D AND 3D DISTORTION MODELS}

The correction of the large geometric distortions which characterize OCT images requires a deformation model that expresses these distortions as a function of the optical path. Indeed, this model will allow the correction of the OCT images both for 2D and 3D scans. To do this, we propose two distortion models for each acquisition mode. Let us consider firstly the source of distortions for only one scanning mirror before formulating both deformation models.

\section{A. Source of distortions}

In case of a 2D acquisition (B-Scan), the OCT light source is redirected to the sample through a single rotating mirror (a galvanometric mirror). An objective lens is used to get a telecentric scanning of the samples as illustrated by Fig. 3. However, if the distance between the lens and the galvo mirror is well adjusted to the focal length $f$ of the objective, 
the scanning is perfectly telecentric (Fig. 3(a)). If the distance is shorter than the focal length, the scanning is divergent (Fig. 3(b)) leading to a convex distortion of the image. In this case, the optical system is equivalent to a scanning without any lens and with a virtual mirror placed very far from the real one $(D>>f)$. On the other hand, if the distance is longer than the focal length, the scanning is convergent (Fig. 3(c)) leading to a concave distortion of the image. In this case, the optical system is equivalent to a scanning without any lens and with a virtual mirror placed very far from the real one but in the opposite direction $(D<0)$.

Since OCT imaging systems usually consist of two separate galvanometric mirrors, the distance from the scan lens cannot be adjusted for both mirrors. The result is that the image is deformed in one or two directions if the focal point falls midway between the mirrors. This last configuration is commonly used as a compromise to reduce the distortion in both directions. This choice explains the convex and concave distortions of 2D scan along perpendicular directions.

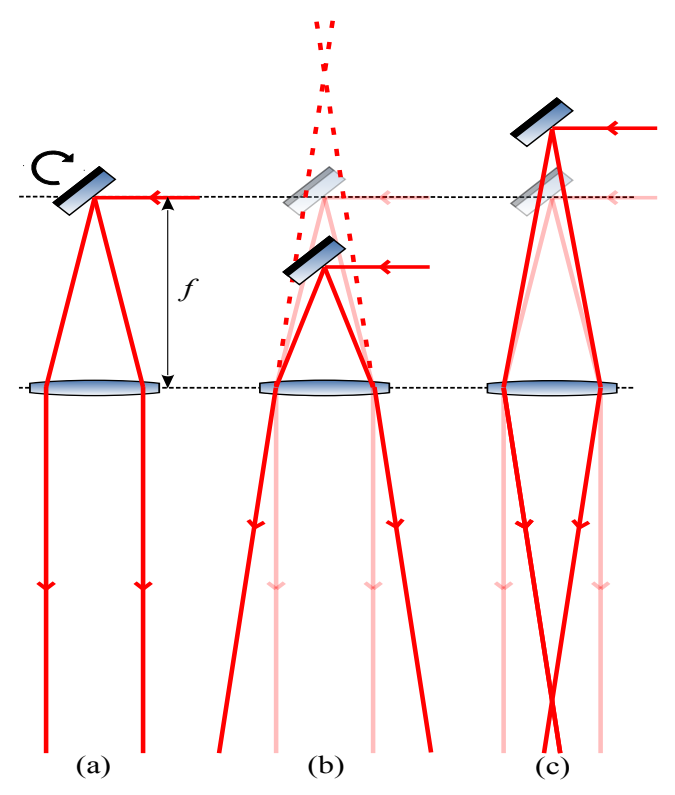

Fig. 3. Illustration of the source of image distortions: (a) the focal point is on the mirror, there is no distortion, (b) the focal point is behind the mirror, the image distortion is convex, (c) the focal point is in front of the mirror, the image distortion is concave.

\section{B. $2 D$ Distortions Model}

2D OCT image acquisition can be ensured either by the mirror 1 or by the mirror 2 . For instance, if we consider the mirror 1, the scanning task is performed by a rotation around the axis $y$ as shown in Fig. 1(a). The whole 2D acquisition scheme is depicted in Fig. 4.

Let us consider the point $O_{c}$ as the rotation centre of the mirror 1 . The set of coordinates $\left(O_{\text {raw }}, x_{\text {raw }}, z_{\text {raw }}\right)$ forms the polar frame $\mathcal{R}_{\text {raw }}$ in which the original (with the geometric distortions) OCT image is expressed. In opposition, the set of coordinates $\left(O_{c}, x_{c}, z_{c}\right)$ represents the Cartesian frame

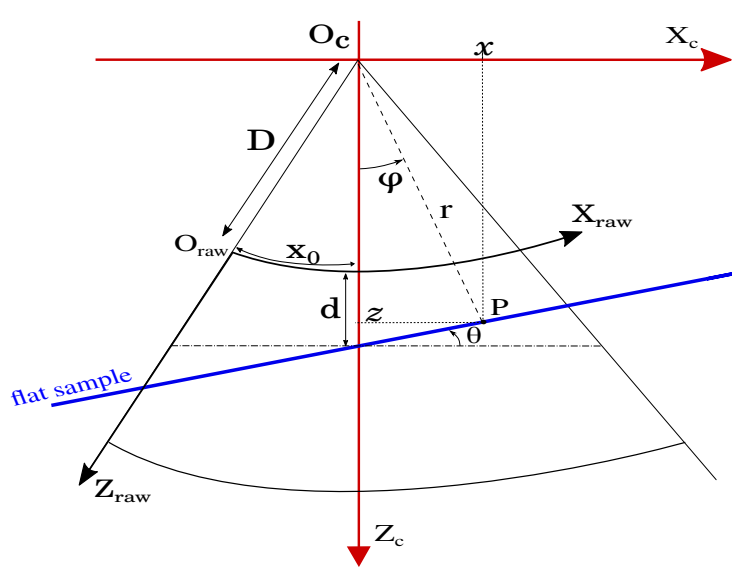

Fig. 4. 2D acquisition model.

$\mathcal{R}_{c}$ in which the corrected image will be projected (forward projection) before a backward projection into $\mathcal{R}_{\text {raw }}$.

The scanning area (OCT field-of-view) is limited by two arcs of circle corresponding to the both minimum and maximum depths to be viewed by the system (Fig. 4). The curvilinear distance from the origin $O_{\text {raw }}$ to the vertical optical axis $\left(O_{c} z\right)$ along an arc of circle is defined by $x_{0}$, when the minimum scanning distance is noticed $D$. It can be highlighted that $x_{O}$ and $D$ are directly related to the internal architecture of the OCT probe, so it can be considered as intrinsic parameters. In opposition, also based on Fig. 4, the extrinsic parameters are the angle $\theta$ and the distance $d$ (along the optical axis $\left(O_{c} z\right)$ ) between the position of the observed sample and the intersection of the $\operatorname{arc} x_{o}$ and $\left(O_{c} z\right)$. Finally, the design of the 2D deformation model consists of the expression of an optical core $z_{\text {raw }}$ (in $\mathcal{R}_{\text {raw }}$ ) as function of $x_{0}, D, \theta$, and $d$.

Let us consider $\mathbf{P}=(x, z)^{\top}$ a physical point of the flat sample which is expressed in $\mathcal{R}_{\text {raw }}$ as follows

$$
\begin{aligned}
x_{\text {raw }} & =x_{0}+\varphi D \\
z_{\text {raw }} & =r-D
\end{aligned}
$$

where $\varphi=\arctan (x, z)$ is the angle between the scanning line (beam position at time $t)$ and the optical axis $\left(O_{c} z\right)$, and $r=\left(x^{2}+z^{2}\right)^{\frac{1}{2}}$ is the distance between $\mathbf{P}$ and the rotation center $O_{c}$ along the scanning line.

Similarly, the expression of $\mathbf{P}$ in $\mathcal{R}_{c a r}$ is given by

$$
\begin{aligned}
& x=r \sin (\varphi) \\
& z=r \cos (\varphi)
\end{aligned}
$$

Furthermore, the equation of the line representing the flat sample, defined in $\mathcal{R}_{\text {car }}$, is written as

$$
z=-x \tan (\theta)+d+D
$$

By introducing (3) and (4) in (5) allows writing

$$
r \cos (\varphi)=-r \sin (\varphi) \tan (\theta)+d+D
$$

Thereby,

$$
r=\frac{d+D}{\cos (\varphi)+\sin (\varphi) \tan (\theta)}
$$


Finally, by mixing (1), (2) and (20), it becomes possible to express the optical core position $z_{\text {raw }}$ (in $\mathcal{R}_{\text {raw }}$ ) as follows

$$
z_{\text {raw }}=\frac{(d+D)}{\cos \left(\frac{x_{r a w}-x_{0}}{D}\right)+\tan (\theta) \sin \left(\frac{x_{r a w}-x_{0}}{D}\right)}-D
$$

\section{3D Distorsions Model}

The 3D deformation model can be considered as an extension of the $2 \mathrm{D}$ one. Indeed, the $3 \mathrm{D}$ acquisition is the combination of a $x z$ and a $y z$ scanning modes of the sample. Consequently, each acquired point $\mathbf{P}$ from the scene is the result of both mirror 1 and mirror 2 scanning effect Fig. 5.

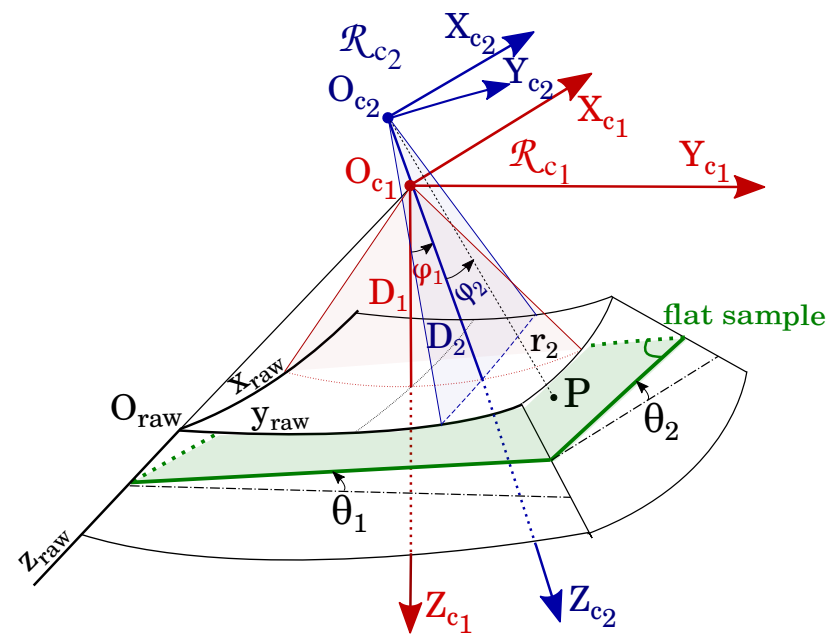

Fig. 5. 3D acquisition model.

Again, let us consider $O_{1}$ and $O_{2}$ the rotation centers of the mirror 1 and 2 , respectively. The polar frame $\mathcal{R}_{\text {raw }}$ becomes a $3 \mathrm{D}$ frame defined by $\left(O_{\text {raw }}, x_{\text {raw }}, y_{\text {raw }}, z_{\text {raw }}\right)$, when there are now two Cartesian frames, i.e., $\mathcal{R}_{c 1}$ $\left(O_{c 1}, x_{c 1}, y_{c 1}, z_{c 1}\right), \mathcal{R}_{c 2}\left(O_{c 2}, x_{c 2}, y_{c 2}, z_{c 2}\right)$ attached to the mirror 1 and 2, respectively. As shown in Fig. 5, the whole 3D deformations model includes the following parameters: intrinsic $\left(x_{0}, y_{0}, D_{1}, D_{2}\right)$, and extrinsic $\left(d, \theta_{1}, \theta_{2}\right)$.

Therefore, the point $\mathbf{P}$ is expressed in $\mathcal{R}_{c 2}$ by

$$
\begin{aligned}
& x_{2}=r_{2} \sin \left(\varphi_{2}\right) \\
& z_{2}=r_{2} \cos \left(\varphi_{2}\right)
\end{aligned}
$$

Then,

$$
\begin{aligned}
\varphi_{2} & =\arctan \left(x_{2}, z_{2}\right) \\
r_{2} & =\sqrt{x_{2}^{2}+z_{2}^{2}}
\end{aligned}
$$

The expression of $\mathbf{P}\left(\widetilde{\mathbf{P}}=(x, y, z, 1)^{\top}\right)$ in $\mathcal{R}_{c 1}$ requires an homogeneous transformation matrix ${ }^{c 1} \mathbf{M}_{\mathbf{c} 2}$ (from $\mathcal{R}_{c 2}$ to $\left.\mathcal{R}_{c 1}\right)$. This transformation can be obtained as follows

$$
{ }^{c 1} \mathbf{M}_{\mathbf{c} 2}=\left[\begin{array}{cccc}
1 & 0 & 0 & 0 \\
0 & \cos \left(\varphi_{1}\right) & \sin \left(\varphi_{1}\right) & \left(D_{1}-D_{2}\right) \sin \left(\varphi_{1}\right) \\
0 & -\sin \left(\varphi_{1}\right) & \cos \left(\varphi_{1}\right) & \left(D_{1}-D_{2}\right) \cos \left(\varphi_{1}\right) \\
0 & 0 & 0 & 1
\end{array}\right]
$$

Furthermore, due the fact that the deformation is convex along $x$-direction and concave along $y$-direction (Fig. 6), it is necessary to introduce a negative sign (-) in the translation part of ${ }^{c 1} \mathbf{M}_{\mathbf{c 2}}$. So, (14) becomes

$$
{ }^{c 1} \mathbf{M}_{\mathbf{c} 2}=\left[\begin{array}{cccc}
1 & 0 & 0 & 0 \\
0 & \cos \left(\varphi_{1}\right) & \sin \left(\varphi_{1}\right) & -\left(D_{1}-D_{2}\right) \sin \left(\varphi_{1}\right) \\
0 & -\sin \left(\varphi_{1}\right) & \cos \left(\varphi_{1}\right) & -\left(D_{1}-D_{2}\right) \cos \left(\varphi_{1}\right) \\
0 & 0 & 0 & 1
\end{array}\right]
$$

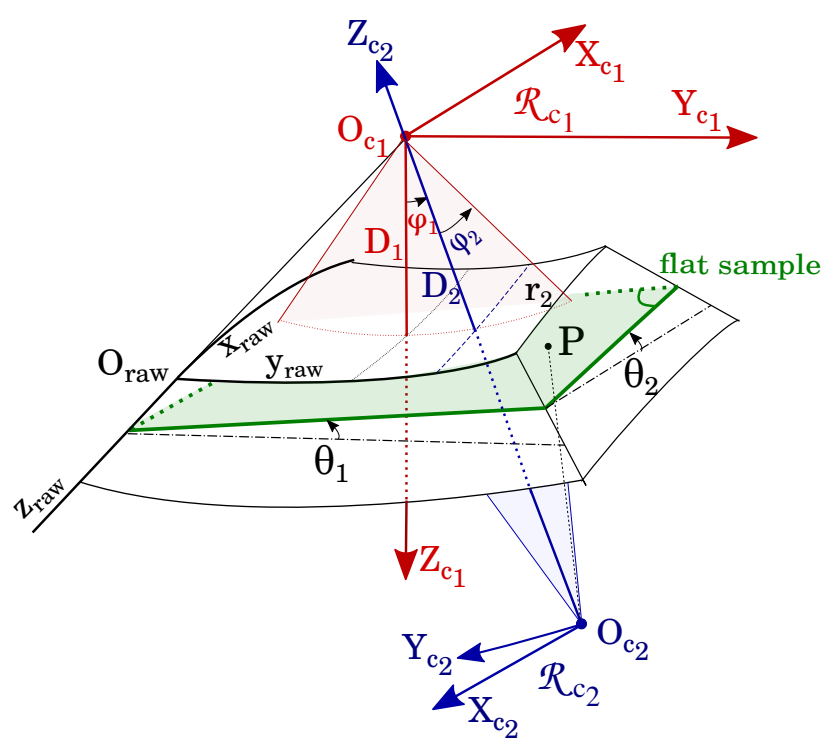

Fig. 6. 3D acquisition scheme when tacking into account the convex and concave forms of the deformation.

In other words, one can write

$$
\widetilde{\mathbf{P}}_{1}={ }^{c 1} \mathbf{M}_{\mathbf{c 2}} \widetilde{\mathbf{P}}_{\mathbf{2}}
$$

where $\widetilde{\mathbf{P}}_{1}$ and $\widetilde{\mathbf{P}}_{2}$ represent the expression of $\widetilde{\mathbf{P}}$ in $\mathcal{R}_{c 1}$ and $\mathcal{R}_{c 2}$, respectively.

The development of (15) using (14) allows writing

$$
\begin{aligned}
& x_{1}=x_{2} \\
& y_{1}=\cos \left(\varphi_{1}\right) y_{2}+\sin \left(\varphi_{1}\right) z_{2}-\left(D_{1}-D_{2}\right) \sin \left(\varphi_{1}\right) \\
& z_{1}=-\sin \left(\varphi_{1}\right) y_{2}+\cos \left(\varphi_{1}\right) z_{2}-\left(D_{1}-D_{2}\right) \cos \left(\varphi_{1}\right.
\end{aligned}
$$

Otherwise, one can write (in $\mathcal{R}_{c 1}$ ) the equation of flat sample (plane) as follows

$$
z_{1}=x_{1} \tan \left(\theta_{1}\right)+y_{1} \tan \left(\theta_{2}\right)+\left(d+D_{1}\right)
$$

By replacing $x_{1}, y_{1}, z_{1}$ by their respective expressions (18) in (19) and by considering $y_{2}=0$ (sample is placed in a horizontal position using a high accurate 6 DOF robotic platform), one obtain

$$
\begin{array}{r}
r_{2}\left(\cos \left(\varphi_{2}\right) \cos \left(\varphi_{1}\right)-\tan \left(\theta_{2}\right) \cos \left(\varphi_{2}\right) \sin \left(\varphi_{1}\right)-\right. \\
\left.\tan \left(\theta_{1}\right) \sin \left(\varphi_{2}\right)\right)=d+D_{1}-\left(\left(D_{2}-D_{1}\right)\left(\cos \left(\varphi_{1}\right)-b \sin \left(\varphi_{1}\right)\right)\right.
\end{array}
$$

Now, it is necessary to express (20) in $\mathcal{R}_{\text {raw }}$. To do this, one start by writing $\mathbf{P}$ in $\mathcal{R}_{\text {raw }}$ as follows

$$
\begin{aligned}
& x_{\text {raw }}=x_{0}+\varphi_{2} D_{2} \\
& y_{\text {raw }}=y_{0}-\varphi_{1} D_{1} \\
& z_{\text {raw }}=r_{2}-D_{2}
\end{aligned}
$$


Thereby, $\varphi_{1}$ and $\varphi_{2}$ are obtained as follows

$$
\begin{aligned}
& \varphi_{2}=\frac{x_{r a w}-x_{0}}{D_{2}} \\
& \varphi_{1}=-\frac{y_{\text {raw }}-y_{0}}{D_{1}} \\
& z_{\text {raw }}=\frac{d+D_{1}-\left(D_{2}-D_{1}\right)\left(\cos \left(\frac{y_{\text {raw }}-y_{0}}{D_{1}}\right)-\tan \left(\theta_{2}\right) \sin \left(\frac{y_{\text {raw }}-y_{0}}{D_{1}}\right)\right)}{\cos \left(\frac{x_{\text {raw }}-x_{0}}{D_{2}}\right)\left(\cos \left(\frac{y_{\text {raw }}-y_{0}}{D_{1}}\right)-\tan \left(\theta_{2}\right) \sin \left(\frac{y_{\text {raw }}-y_{0}}{D_{1}}\right)\right)-\tan \left(\theta_{1}\right) \sin \left(\frac{x_{\text {raw }}-x_{0}}{D_{2}}\right)}-D_{2}
\end{aligned}
$$

Finally, introducing $\varphi_{1}$ and $\varphi_{2}$ in (20) and considering $z_{\text {raw }}$ formulation in (23), allows defining the expression of

\section{EXPERIMENTAL VALIDATION}

\section{A. OCT Imaging Device}

OCT imaging system (a Telesto-II 1325nm spectral domain OCT) from THORLABS (Fig. 7), is used to validated the proposed distortions models. It is characterized by $5.5 \mu \mathrm{m}$ and $7 \mu \mathrm{m}$ axial and lateral resolutions, respectively, and provides up to $3.5 \mathrm{~mm}$ of depth. The Telesto-II allows a maximum field-of-view of $10 \times 10 \times 3.5 \mathrm{~mm}^{3}$ with a maximum A-Scan (optical core) acquisition rate of $76 \mathrm{kHz}$. The studied sample can also be viewed by a conventional CCD camera (with a resolution of $640 \times 480$ pixels at 25 frame per second) rigidly fixed to the OCT probe.

In order to evaluate the proposed deformation models, we implemented three scenarios: 1) compensation of the geometric distortions in B-Scan images, 2) in 3D OCT images and 3) using biological samples in both $2 \mathrm{D}$ and $3 \mathrm{D}$ acquisition modes.

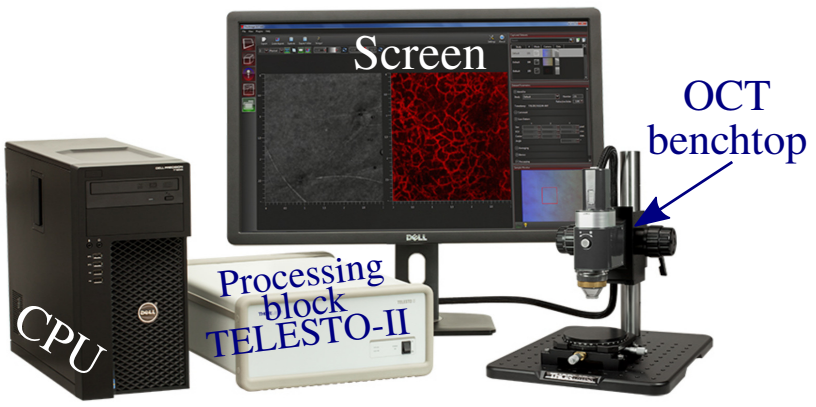

Fig. 7. Global view of the OCT acquisition setup.

\section{B. 2D Parameters Estimation}

Calibration

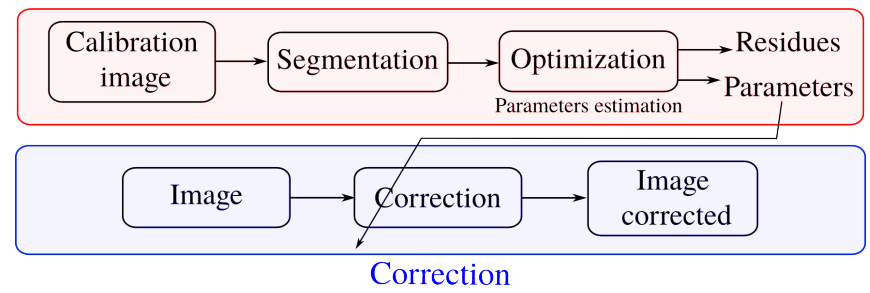

Fig. 8. Scheme of the whole OCT image correction algorithm.
To validate the proposed methods, we start by using the perfect flat sample shown in Fig. 2(a). This sample allows both computing the intrinsic and extrinsic parameters (in 2D and $3 \mathrm{D}$ acquisition modes) and performing the first ruthground verification. Fig. 8 presents the different steps that contained our method for compensating the whole distortions parameters. As can be noticed, it remains one step which is the optimization process. The later consists of minimizing the error between the real deformation computed (using a segmentation algorithm) from the OCT image and that of the theoretical model. The problem is relatively simple, thus we use least squares criterion $\mathcal{J}_{2 D}$ given by

$$
\mathcal{J}_{2 D}=\sum_{i}\left(z_{\text {raw }}(i)-\hat{z}_{\text {raw }}(i)\right)^{2}
$$

where $z_{\text {raw }}(i)$ is $i-t h$ depth computed from the OCT image (here a B-Scan) and the corresponding depth $\hat{z}_{\text {raw }}(i)$ provided by the model (8).

The result of the optimization process is the estimated geometric distortions parameters, i.e., $D, x_{0}, d$ and $\theta$. Fig. 9(a-b) illustrates the identification of the 2D deformation model in two examples. As can be noticed the measured deformation superposes accurately the computed one (using our method). The root means square (RMS) error between both estimated and computed curves is $e_{r m s}=1.06$ pixels with a standard deviation (STD) of $e_{s t d}=1.1$ pixels. The whole process takes only 0,8 second (under a non-optimized MATLAB implementation).
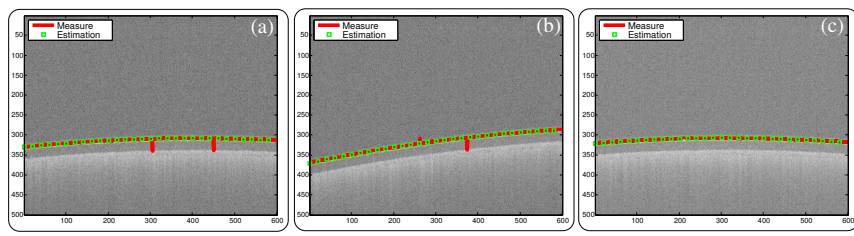

Fig. 9. Validation of the 2D deformation model.

Such as for the projective cameras calibration, to avoid the coupling effect between the intrinsic and extrinsic parameters, it is necessary to acquire several images in different positions and orientations during the identification process. The final numerical values of the $2 \mathrm{D}$ model are

$$
\left[D(p i x), x_{0}(p i x), d(p i x), \theta(d e g)\right]=[4372,308,315,0.138]
$$


1) 3D Parameters Estimation: Now, let us estimate the parameters of the 3D deformation model. Similarly, to the 2D one, we use a quadratic criterion-based optimization method. The new criterion $\mathcal{J}_{3 D}$ is given by

$$
\mathcal{J}_{3 D}=\sum_{i} \sum_{j}\left(z_{\text {raw }}(i, j)-\hat{z}_{\text {raw }}(i, j)\right)^{2}
$$

where $z_{\text {raw }}(i, j)$ is $i, j-t h$ depth computed from the 3D OCT image and $\hat{z}_{\text {raw }}(i, j)$ the corresponding depth estimated thanks to our model (III-C).
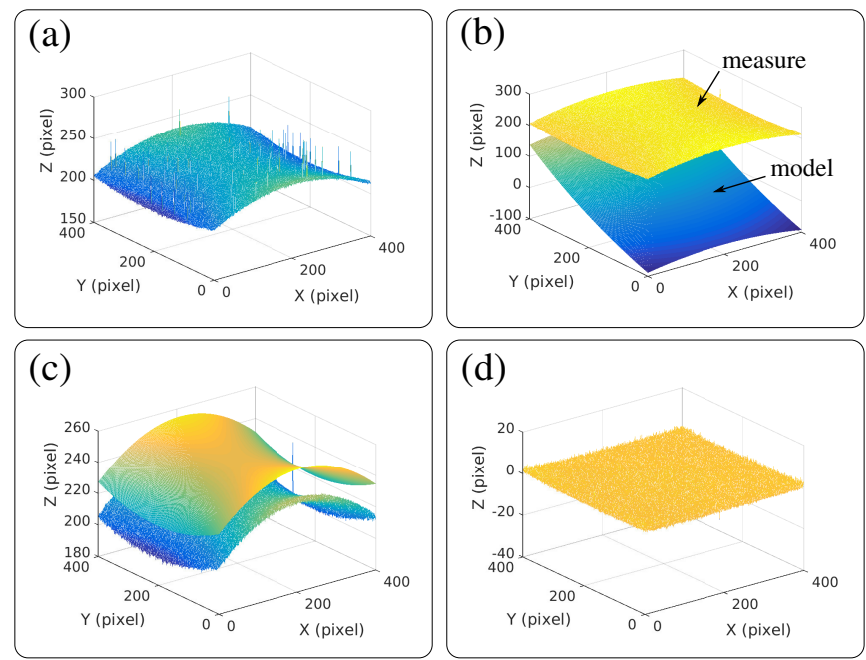

Fig. 10. Validation of the 3D deformation model: (a) the segmented curve, (b) both the segmented and the estimated curves before optimization, (c) after optimization with a voluntary added offset in $z$ for a better illustration, and (d) without this offset.

The same flat sample (used for the 2D case) is used to demonstrate the operating of the 3D deformations model (Fig. 10). More precisely, Fig. 10(a) depicts the flat sample (after segmentation) as viewed by the OCT device while Fig. 10(b) shows both the segmented shape and the estimated one using the 3D model (before the optimization process). Therefore, using the 3D geometric distortions parameters provided by our model, we obtain the shape illustrated in Fig. 10(c). Note that there is an offset in $z$ between the segmented and estimated shapes. This is voluntary for a better illustration of the results. When removing this offset, both surfaces are perfectly superimposed (Fig. 10(d) shows the error between both surfaces). The computed RMS error between both surfaces is estimated to $e_{r m s}=0.78$ pixel with a STD of $e_{\text {std }}=0.80$ pixel.

The numerical values of the 3D model parameters (intrinsic + extrinsic), computed in 5.35 seconds, are:

$\left[D_{1}(\right.$ pix $), D_{2}($ pix $), x_{0}($ pix $), y_{0}($ pix $), d($ pix $\left.), \theta_{1}(d e g), \theta_{2}(d e g)\right]=$ [1937.4, -782.1, 227.4, 209.4, 310.6, -0.001, -0.0002]

\section{C. $2 D$ et $3 D$ Images Correction}

The last step of the geometric distortions process (as shown in Fig. 8) is the images correction. This allows transforming the acquired OCT image from $\mathcal{R}_{\text {raw }}$ towards
$\mathcal{R}_{c}$ by taking into account the geometric distortions during the projection. This geometric transformation is based on the forward and backward mapping [11] method (Fig. 11) .
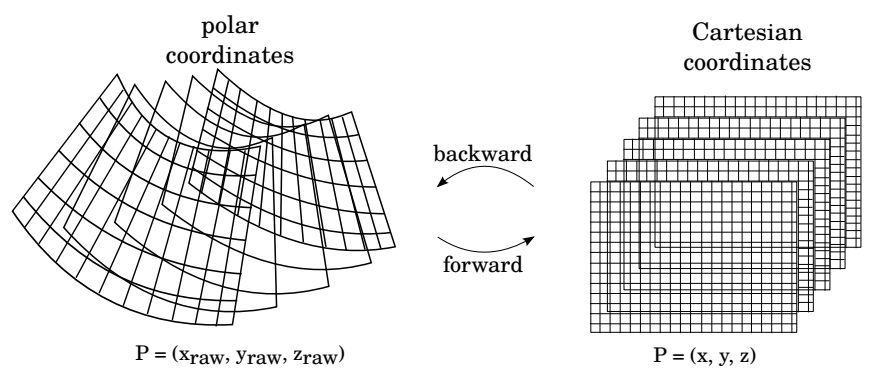

Fig. 11. Forward and backward mapping method.

In fact, the geometrical transformation is applied to the plane $\left(O_{\text {raw }}, x_{\text {raw }}, z_{\text {raw }}\right)$ towards the plane $(O, x, z)$ (i.e., forward mapping). Afterwards, an inverse transformation (i.e., backward mapping) is applied to express the corrected coordinates of each pixel into the polar frame $\mathcal{R}_{\text {raw }}$. Finally, the obtained 2D OCT image is shown in Fig. 12. As can be highlighted the corrected image (Fig. 12(b)) shows horizontal lines which correspond to the reality of the observed scene (flat and horizontal sample) unlike to the initial image which shows curved lines (Fig. 12(a)).
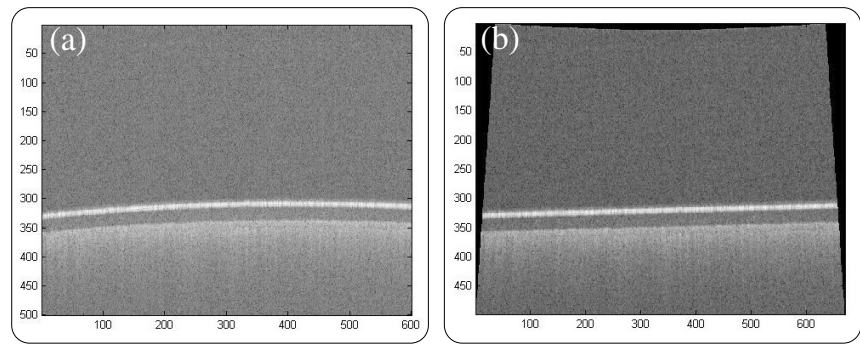

Fig. 12. 2D image correction: (a) with distortions and (b) without distortions.

The method is applied (using the 3D deformations model) for 3D OCT images correction. As can be seen in Fig. 13, it is possible to compensate accurately the different geometric distortions on an OCT volume. Fig. 13(a) and Fig. 13(b) depict OCT volume before and after the geometric distortions compensations.

\section{Validation on a Biological Sample}

As has been mentioned in this paper, OCT images (2D or 3D) can be considered as substitution means to establish a diagnosis, for example in ophthalmology where the traditional physical biopsy is to be avoided. A reliable optical biopsybased diagnosis requires OCT images which must reflect perfectly the reality of the studied sample. The proposed models to compensate the different geometric distortions has been validated in biological sample (here a $f l y$ ). As can be seen in Fig. 14, the images are corrected providing realistic optical biopsies. 

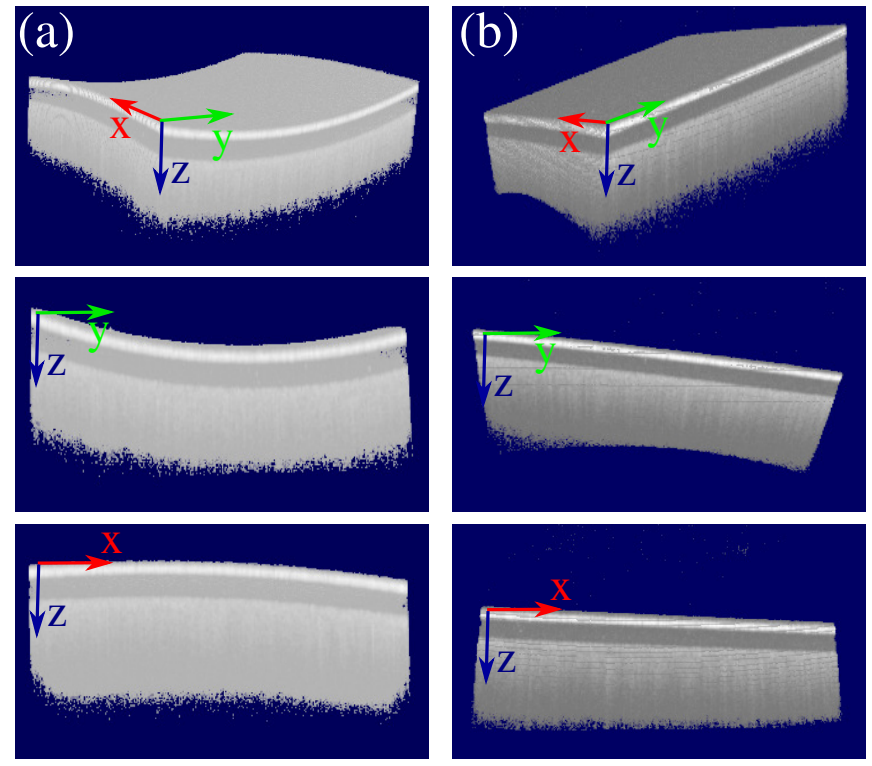

Fig. 13. Validation on 3D OCT image: (a) $y z$ initial OCT images (extracted from an OCT volume), (b) the corrected $y z$ images, (c) $x z$ initial images and (d) the $x z$ corrected ones.

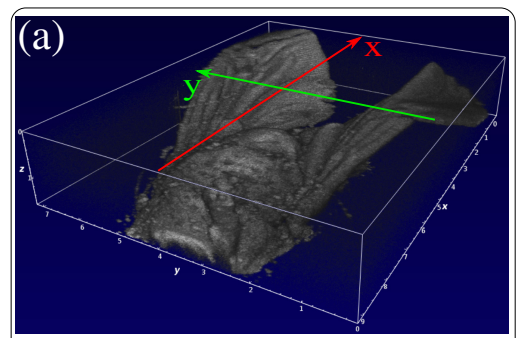

3D-Scan of a fly
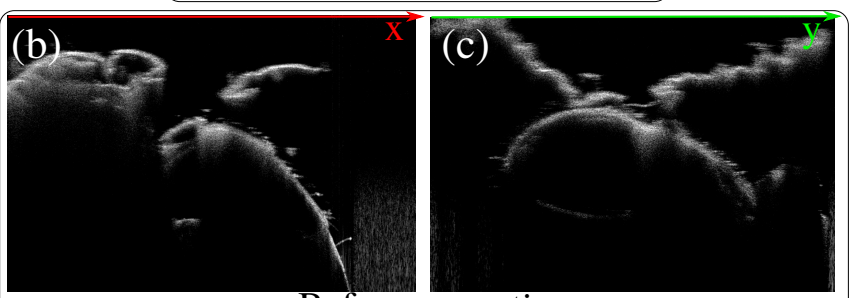

Before correction

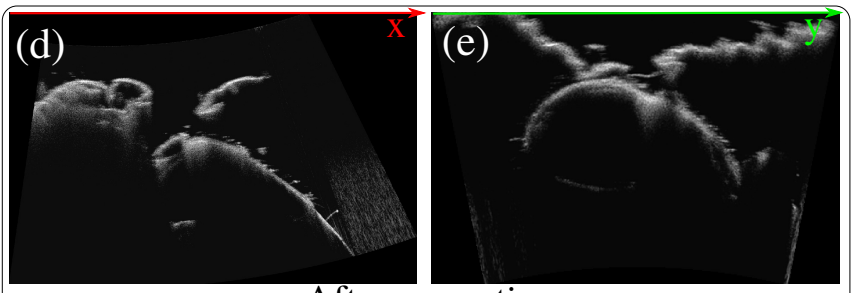

After correction

Fig. 14. Validation on a biological sample (a fly): (a) initial 3D OCT image, (b) $x z$ OCT image before correction, (c) $y z$ OCT image before correction, (d) $x z$ corrected image and (e) $y z$ corrected image.

\section{CONCLUSION}

In this paper, it was proposed two geometric models for OCT images corrections. These methods use a simple 2D flat sample (without any visual markers or known structures) placed horizontally on the sample holder. OCT images (2D or 3D) are characterized by large geometric distortions (spherical, angular and fan) which make it difficult to reliable diagnosis use in medical field. To tackle this problem, it was proposed to describe the OCT images (2D and 3D) acquisition through geometric models which include the intrinsic as well as the extrinsic parameters of the OCT device. Using the developed models, it was demonstrated (experimentally) that it becomes possible to compensate efficiency the geometric distortions in both 2D and 3D images.

\section{REFERENCES}

[1] Z. Zhang, "A flexible new technique for camera calibration," IEEE Trans. on Pattern Analysis and Machine Intelligence, vol. 22, no. 11, pp. 1330-1334, 2000.

[2] R. Hartley and A. Zisserman, Multiple view geometry in computer vision. Cambridge University Press, 2003.

[3] M. Ourak, B. Tamadazte, and N. Andreff, "Partitioned CameraOCT based 6 DOF Visual Servoing for Automatic Repetitive Optical Biopsies", 'IEEE/RSJ Int. Conference on Robotics and Intelligent Systems, South Korea, DOI:10.1109/IROS.2016.7759364, 2016.

[4] M. Ourak, A. De Simone, B. Tamadazte, G. J. Laurent, A. Menciassi, and N. Andreff, "Automated in-plane oct-system positioning towards repetitive optical biopsies," IEEE Int. Conference on Robotics and Automation, Sweden, pp. 4186-4191, 2016.

[5] V. Westphal, A. Rollins, S. Radhakrishnan, and J. Izatt, "Correction of geometric and refractive image distortions in optical coherence tomography applying fermat's principle," Optics Express, vol. 10, no. 9, pp. 397-404, 2002.

[6] S. Van der Jeught, J. A. Buytaert, A. Bradu, A. G. Podoleanu, and J. J. Dirckx, "Large-volume optical coherence tomography with real-time correction of geometric distortion artifacts," arXiv:1212.1595, 2012.

[7] J. D. Diaz, M. Rahlves, O. Majdani, E. Reithmeier, and T. Ortmaier, "A one step vs. a multi step geometric calibration of an optical coherence tomography," in SPIE BiOS. Int. Society for Optics and Photonics, 2013, pp. $85730 \mathrm{D}-85730 \mathrm{D}$.

[8] D. Wang, P. Liang, S. Samuelson, H. Jia, J. Ma, and H. Xie, "Correction of image distortions in endoscopic optical coherence tomography based on two-axis scanning mems mirrors," Biomedical Optics Express, vol. 4, no. 10, pp. 2066-2077, 2013.

[9] D. Huang, E. A. Swanson, C. P Lin, J. S Schuman, et al. "Optical coherence tomography", Science. 254 (5035): 1178?81, 1991.

[10] J. A. Izatt, M. A. Choma, and A.-H. Dhalla, "Theory of optical coherence tomography," Optical Coherence Tomography: Technology and Applications, pp. 65-94, 2015.

[11] G. Wolberg, Digital image warping. IEEE Computer Society Press Los Alamitos, CA, 1990, vol. 10662. 